\title{
Cyr61 decreases Cytarabine chemosensitivity in acute lymphoblastic leukemia cells via NF-kB pathway activation
}

\author{
YINGPING CAO $^{1 *}$, CONGLIAN WU $^{1,2^{*}}$, YANFANG SONG $^{3}$, ZHEN LIN $^{1}$, YANLI KANG $^{1}$, PINGXIA LU $^{1}$, \\ CHENQING ZHANG $^{1}$, QINGHUA HUANG ${ }^{1}$, TAISEN HAO ${ }^{4}$, XIANJIN ZHU ${ }^{1 *}$ and JIANDA HU ${ }^{1,5^{*}}$ \\ ${ }^{1}$ Department of Laboratory Medicine, Fujian Medical University Union Hospital, Fuzhou, Fujian 350001; \\ ${ }^{2}$ Department of Laboratory Medicine, The First Hospital of Quanzhou Affiliated to Fujian Medical University, Quanzhou, \\ Fujian 362000; ${ }^{3}$ Department of Laboratory Medicine, Clinical Laboratory, The Affiliated People's Hospital of Fujian \\ University of Traditional Chinese Medicine, Fuzhou, Fujian 350001, P.R. China; ${ }^{4}$ Department of Cancer Biology, \\ Beckman Research Institute, City of Hope, Duarte, CA 91010, USA; ${ }^{5}$ Fujian Medical University Union Hospital, \\ Fujian Institute of Hematology, Fujian Province Key Laboratory of Hematology, Fuzhou, Fujian 350001, P.R. China
}

Received August 9, 2018; Accepted November 11, 2018

DOI: $10.3892 /$ ijmm.2018.4018

\begin{abstract}
Elevated Cyr61 levels have been reported in various malignancies. Elevation of Cyr61 protein levels contributes to the proliferation, metastasis, and chemotherapy resistance of malignant cells. Previously, it was discovered that Cyr61 is elevated in both the plasma and the bone marrow supernatants of patients with acute lymphoblastic leukemia (ALL), promoting ALL cell survival. However, the role of Cyr61 in the chemotherapeutic resistance of ALL cells remains unknown. The aim of the current study was to investigate the role of Cyr61 in regulating ALL cell chemosensitivity to Ara-C. It was found that Cyr61 is overexpressed in bone marrow mononuclear cells from patients with ALL. Increased Cyr61 effectively decreased Ara-C-induced apoptosis of ALL cells, and its function was blocked by the use of the anti-Cyr61 monoclonal antibody 093G9. Furthermore, Cyr61 increased the level of Bcl-2 in Ara-C-treated ALL cells. Mechanistically, it was shown that Cyr61 affected ALL cell resistance to Ara-C partially via the $\mathrm{NF}-\kappa \mathrm{B}$ pathway. Taken together, the present study is the first, to the best of our knowledge, to reveal that Cyr61 is involved in ALL cell resistance through the NF- $\mathrm{B}$ pathway. The findings support a functional role for Cyr61 in promoting chemotherapy resistance, suggesting that targeting
\end{abstract}

Correspondence to: Dr Xianjin Zhu or Dr Jianda Hu, Department of Laboratory Medicine, Fujian Medical University Union Hospital, 29 Xinquan Road, Fuzhou, Fujian 350001, P.R. China

E-mail: zxj5027667@163.com

E-mail: drjiandahu@163.com

*Contributed equally

Key words: Cyr61, acute lymphoblastic leukemia, Ara-C, NF-кB signaling pathway
Cyr61 directly or its relevant effector pathways may improve the clinical responses of patients with ALL.

\section{Introduction}

Acute lymphoblastic leukemia (ALL) is an aggressive hematological malignancy that is mainly diagnosed in children. ALL arises from the malignant transformation of $\mathrm{T}$ or $\mathrm{B}$ progenitor cells in the bone marrow (BM) $(1,2)$. Current multi-agent chemotherapy regimens are highly effective in patients with newly-diagnosed ALL (3); however, a significant number of patients relapsed due to chemotherapy failure. Resistance to chemotherapeutic agents is one of the major obstacles for the successful treatment of ALL $(3,4)$.

Previous studies showed that the mechanism of resistance to chemotherapeutics agents is a complex network involving multiple cellular and molecular mechanisms. In ALL, the BM microenvironment provides growth and survival signals that may confer resistance to chemotherapy (5-7). Increasing evidence suggest that soluble factors in BM, including extracellular matrix molecules, cytokines, and chemokines such as osteopontin, CXCL12, and interleukin-6 $(8,9)$, provide a permissive environment for leukemogenesis and contribute to drug resistance $(10,11)$. Thus, studies on soluble factors in BM provide a better understanding of the drug resistance of ALL and facilitate the design of new treatments.

Cyr61/CCN1 is a secreted extracellular matrix (ECM) protein, which is important for cell proliferation, survival, adhesion, migration, and differentiation (12). As a secreted protein, the role of Cyr61 has been extensively investigated in solid tumors, with multiple studies showing that Cyr61 positively regulates tumor cell growth and metastasis (13-16). More and more studies have shown that Cyr61 confers on malignant cells resistance to chemotherapeutic drugs in breast cancer, ovarian cancer, prostate carcinoma and pancreatic ductal adenocarcinoma (17-20). Notably, Cyr61 is also involved in stroma-induced chemo-resistance in acute myeloid leukemia (AML) (21). In 2016, it was found that the levels of 
Cyr61 are elevated in the plasma and BM supernatants from patients with ALL compared with in samples from healthy donors. It was also shown that increased Cyr61 promotes ALL cell survival (22). However, whether Cyr61 is involved in ALL cell resistance to chemotherapeutic drugs has not yet been explored.

In the present study, it was revealed that Cyr61 is highly expressed in $\mathrm{BM}$ mononuclear cells (BMMNCs) from patients with ALL compared with those from healthy donors. Furthermore, the role of Cyr61 in the chemotherapeutic sensitivity of ALL cells was determined. Given that Cytosine arabinoside (Ara-C) is one of the most important chemotherapeutic agents used to treat both children and adults with acute leukemia (3), our study used Ara-C to evaluate the role of Cyr61 in the chemotherapy resistance of ALL cells. The present study found that Cyr61 could protect ALL cells from Ara-C-induced apoptosis and that its effectiveness might be partially due to the activation of the NF- $\mathrm{BB}$ pathway. Furthermore, it was found that blocking the bioactivity of Cyr61 with an anti-Cyr61 antibody 093G9 could improve the ALL cell response to Ara-C. Therefore, the results indicated that Cyr61 may act as a chemoprotective factor for ALL cells, and that targeting Cyr61 directly, or its relevant effector pathways, might improve the clinical responses of patients with ALL.

\section{Materials and methods}

Reagents and chemicals. Recombinant human (rh) Cyr61 was obtained from PeproTech, Inc., (Rocky Hill, NJ, USA), dissolved in PBS to a stock concentration of $1 \mathrm{mg} / \mathrm{ml}$ and stored at $80^{\circ} \mathrm{C}$ until used. Ara-C was purchased from Sigma-Aldrich (Merck KGaA, Darmstadt, Germany), dissolved in DMSO to a stock concentration of $0.5 \mathrm{mM}$ and stored at $-20^{\circ} \mathrm{C}$ until used. A mouse anti-human Cyr61 monoclonal antibody (093G9) and a PEGFP-Cyr61 plasmid were kindly gifted by Dr Ningli Li (Shanghai Jiao Tong University School of Medicine, Shanghai, China). Rabbit anti-human monoclonal antibodies $(\mathrm{mAb})$ against GAPDH (1:1,000; cat. no. 8884S), Bcl-2 (1:1,000; cat.

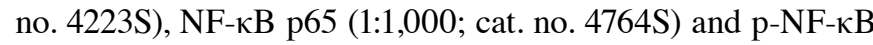
p-p65 (1:1,000; cat. no. 3033S) used were all purchased from Cell Signaling Technology, Inc. (Danvers, MA, USA). An HRP-linked anti-rabbit IgG and HRP-linked anti-mouse IgG were purchased from Cell Signaling Technology, Inc. Pyrrolidine dithiocarbamate (PDTC; special inhibitor of NF- $\mathrm{kB}$ activation) was purchased from Sigma-Aldrich (Merck KGaA).

Patients and specimens. All bone marrow (BM) aspirate samples were obtained from Fujian Medical University Union Hospital (Fuzhou, China) from July 2015 to October 2017. To analyze the level of Cyr61 in the BM mononuclear cells (BMMNCs) from patients with ALL, BM samples from newly diagnosed, non-treated patients with ALL (range, 6-37 years, $\mathrm{n}=8$ ) and healthy donors (range, 16-34, $\mathrm{n}=6$ ) were collected (Table I) and enriched for BMMNCs using histopaque gradient centrifugation (density $1.077 \mathrm{~g} / \mathrm{ml}$; Sigma-Aldrich; Merck $\mathrm{KGaA}$ ) according to the manufacturer's instructions. The BM supernatant samples were obtained after centrifugation of the total BM aspirates of consecutive patients with ALL and stored at $-80^{\circ} \mathrm{C}$ until used. These studies were performed in accordance with the ethical guidelines under the protocols approved by the Institutional Medical Ethics Review Board of the Fujian Medical University Union Hospital. Informed consent was obtained from all individual participants included in the study.

Cell lines and culture conditions. Leukemia cell lines Jurkat and Nalm-6 cells were kindly provided by Dr Qiang Chen (Shanghai Jiao Tong University School of Medicine) and were maintained in RPMI-1640 medium (HyClone; GE Healthcare Life Sciences, Logan, UT, USA) supplemented with $10 \%$ fetal bovine serum (FBS; Gibco; Thermo Fisher Scientific, Inc., Waltham, MA, USA), $100 \mathrm{U} / \mathrm{ml}$ penicillin and $100 \mathrm{mg} / \mathrm{ml}$ streptomycin at $37^{\circ} \mathrm{C}$ and $5 \% \mathrm{CO}_{2}$.

Evaluation of Cyr61's effect on ALL cell chemosensitivity and antibody neutralization assays. To address the effect of BM-derived Cyr61 on the chemotherapeutic sensitivity to Ara-C of ALL cells, ALL cells were cultured using BM supernatants from patients with ALL in which overexpressed Cyr61 had been blocked with $1,000 \mathrm{pg} / \mathrm{ml}$ 093G9. A murine isotype-matched antibody served as the control. Cells were then treated with $1 \mu \mathrm{M}$ Ara-C for $24 \mathrm{~h}$ and apoptotic cells were quantified by Annexin V-FITC and PI double-staining kit (BD Biosciences, San Jose, CA, USA).

Next, to explore rhCyr61's effect on chemosensitivity in ALL, Jurkat $\left(5 \times 10^{6}\right.$ cells $\left./ \mathrm{ml}\right)$ and Nalm- $6\left(1 \times 10^{7}\right.$ cells $\left./ \mathrm{ml}\right)$ cells were seeded into 24-well plates (CoStar, Cambridge, MA, USA) and maintained in RPMI-1640 medium with 5\% FBS. Jurkat and Nalm-6 were pre-incubated with rhCyr61 at different concentrations for $24 \mathrm{~h}$ followed by treatment with $1 \mu \mathrm{M}$ Ara-C. After incubation for $24 \mathrm{~h}$, cell apoptosis was analyzed by Annexin V-FITC and PI double-staining kit (BD Biosciences).

For the antibody blocking assay, rhCyr61 was pre-incubated with a mouse anti-Cyr61 mAb (093G9) for $2 \mathrm{~h}$ prior to adding to cell culture. A murine isotype-matched antibody served as a control. After incubation for $24 \mathrm{~h}$, Jurkat and Nalm- 6 cells were treated with $1 \mu \mathrm{M}$ Ara-C for another $24 \mathrm{~h}$. Cell apoptosis was analyzed using an Annexin V-FITC and PI Double-Staining kit (BD Biosciences).

Apoptosis assay. Cell apoptosis was measured according to the manufacturer's instruciton (BD Biosciences). The percentages of cell apoptosis (FITC-positive) were analyzed by flow cytometry using BD FACS Canto II flow cytometer and BD FACSDiva 6.0 software (BD Biosciences).

Transfection and Ara-C induced apoptosis assay. To construct Cyr61-overexpressing ALL cell models, Jurkat and Nalm- 6 cells were transfected with PEGFP-Cyr61 (the plasmid carrying Cyr61 cDNA) using Lipofectamine 2000 (Invitrogen; Thermo Fisher Scientific, Inc.) in serum-free RPMI-1640 according to the manufacturer's instructions. ALL cells were transfected with PEGFP-N3 (vector only) to generate matched control cells. After transfection, cells were washed and then incubated in RPMI-1640 medium with $10 \%$ FBS for $48 \mathrm{~h}$. For the apoptosis assay, the transfected cells were treated with $1 \mu \mathrm{M}$ Ara-C for $24 \mathrm{~h}$. The cells were then washed in 1X PBS and stained with Annexin V-PE 
Table I. Clinical characteristics of patients with ALL included in the present study.

\begin{tabular}{lcc}
\hline Characteristics & ALL $(\mathrm{n}=8)$ & Control $(\mathrm{n}=6)$ \\
\hline Sex & & \\
Male & 3 & 3 \\
Female & 5 & $21.63 \pm 7.25$ \\
Age (years) & $20.15 \pm 12.78$ & \\
Diagnosis & & \\
T-ALL & 2 & \\
B-ALL & 6 &
\end{tabular}

(BD Biosciences) according to the manufacturer's instructions. The percentages of apoptotic Jurkat or Nalm-6 cells (PE-positive) were subsequently analyzed by flow cytometry using a BD FACS Canto II flow cytometer and BD FACSDiva software 6.0 (BD Biosciences).

Real-time PCR. Jurkat and Nalm-6 were treated with Ara-C $(1 \mu \mathrm{M})$ with or without rhCyr61 (100 ng/ml for Jurkat and $1,000 \mathrm{ng} / \mathrm{ml}$ for Nalm-6) for $8 \mathrm{~h}$ before RNA extraction. Total RNA was extracted from specimens using a TriPure Isolation Reagent (Roche Diagnostics, Indianapolis, IN, USA) according to the manufacturer's instructions. Total RNA $(1 \mu \mathrm{g})$ was reverse transcribed into first strand cDNA using the RevertAid ${ }^{\mathrm{TM}}$ First Strand cDNA Synthesis kit (Thermo Fisher Scientific, Inc.). Briefly, $1 \mu \mathrm{l}$ of $50 \mu \mathrm{M}$ oligo (dT)20 and $1 \mu \mathrm{l}$ of $10 \mathrm{mM}$ dNTPs mix were added to the RNA, and the volume was adjusted to $11 \mu \mathrm{l}$ using RNase-free water. mRNA was converted to cDNA according to the kit manufacturer's instructions. Real-time PCR was performed using SYBR $^{\circledR}$-Green Master Mix (Applied Biosystems; Thermo Fisher Scientific, Inc.) according to the manufacturer's instructions. The primers used in this study were as follows: Bcl-2, forward, CTGGTGGGAGCTTGCATCAC; Bcl-2, reverse, ACAGCCTGCAGCTTTGTTTC; BCL-xL, forward, TCA GGCTGCTTGGGATAAAGAT; BCL-xL, reverse, AGAGGC TTCTGGAGGACATTTG; Bax, forward, TGGAGCTGC AGAGGATGATTG; Bax, reverse, CCAGTTGAAGTTGCC GTCAGA; XIAP, forward, TTGAGGAGTGTCTGGTAAG; XIAP, reverse, CCATTCGTATAGCTTCTTGT; GAPDH, forward, CACATGGCCTCCAAGGAGTA; GAPDH, reverse, TGAGGGTCTCTCTCTTCCTCTTGT. GAPDH was used as an internal control, and the relative expression of each mRNA was analyzed using the $2^{-\Delta \Delta C t}$ method (23).

Probing of signaling pathways involved in Cyr61-enhanced resistance to Ara-C in ALL cells. Approximately $2.5 \times 10^{5}$ cells/well Jurkat, $5 \times 10^{6}$ cells/well Nalm- 6 were plated in 24-well plates with $500 \mu \mathrm{l}$ RPMI-1640 containing 5\% FBS. Next, $4 \mu \mathrm{M}$ PDTC (an inhibitor of the NF- $\mathrm{BB}$ pathway) was added to the plates along with rhCyr61 (100 ng/ml for Jurkat and 1,000 ng/ml for Nalm-6). After pre-incubation for $24 \mathrm{~h}$ the plates were treated with $1 \mu \mathrm{M}$ Ara-C. After incubation for $24 \mathrm{~h}$, cell apoptosis was measured using an Annexin V-FITC and PI double-staining kit (BD Biosciences).
Western blot analysis. Protein immune blotting was performed as described previously (24). Briefly, cells were lysed with cell lysis buffer (Beyotime Institute of Biotechnology, Haimen, China) according to the manufacturer's instructions and protein concentration was detected using a Bio-Rad Protein Assay kit (Bio-Rad, Hercules, CA, USA). Proteins were separated using a pre-cast $10 \%$ SDS-PAGE gel and subsequently transferred onto a PVDF membrane (EMD Millipore, Billerica, MA, USA) at $90 \mathrm{~V}$ for $90 \mathrm{~min}$. The blots were blocked with $5 \% \mathrm{BSA}$ for $1 \mathrm{~h}$ at room temperature with gentle shaking, and then, respectively, probed with rabbit anti-human mAbs against GAPDH and Bcl-2 (1:1,000) or mouse anti-human mAbs against Cyr61, with gentle shaking overnight at $4^{\circ} \mathrm{C}$ overnight and subsequently rinsed with PBS. The blots were then incubated with an HRP-linked anti-rabbit $\operatorname{IgG}(1: 2,000)$ or HRP-linked anti-mouse IgG $(1: 2,000)$ for $1 \mathrm{~h}$ at room temperature with gentle shaking. After three rounds of washing with PBS, the target proteins were examined with an ECL system (EMD Millipore) and visualized with autoradiography film. The housekeeping protein GAPDH was selected as an internal control for equal protein loading.

Statistical analysis. Unless otherwise indicated, the results are expressed as the mean \pm standard error of the mean (SEM). Statistical analyses were performed with SPSS software version 18.0 (SPSS Inc., Chicago, IL, USA). The difference in Cyr61 expression between patients with ALL and healthy donors was analyzed by the non-parametric Mann-Whitney $\mathrm{U}$ test. Statistical comparisons of means between two groups were analyzed using the Student's t-test. Comparisons among multiple groups were analyzed using one-way ANOVA with a post hoc SNK test for comparisons. $\mathrm{P}<0.05$ was considered to indicate a statistically significant difference.

\section{Results}

Level of Cyr61 expression in ALL cell lines and ALL bone marrow samples. It was previously observed that Cyr61 is elevated in both the plasma and BM supernatants from patients with ALL. In this study, the levels of Cyr61 protein were examined in the BMMNCs derived from eight patients with ALL and six healthy donors via western blotting, and the results showed that Cyr61 levels in the BMMNCs derived from patients increased to varying levels compared with those derived from healthy donors (Fig. 1A). Further analysis showed that Cyr61 protein was expressed in both Jurkat and Nalm-6 cells, and that the level of Cyr61 protein in Jurkat cells was approximately 1.5-fold higher than in Nalm-6 cells $(\mathrm{P}<0.01$, Student's t-test; Fig. 1B). Taken together, these data indicate that the level of Cyr61 is upregulated in ALL bone marrow samples, and that ALL cell lines (Jurkat and Nalm-6) also express the Cyr61 protein.

Cyr61 effectively decreases Ara-C-induced apoptosis in ALL cells. Previous studies have shown that Cyr61 is involved in drug resistance by decreasing chemotherapeutic drug-induced apoptosis in ovarian cancer, breast cancer and acute myeloid leukemia cells $(21,25,26)$. To explore the role of Cyr61 in the drug resistance of ALL, Jurkat (T-ALL cell lines) and Nalm-6 (B-ALL cell lines) cells were incubated with BM supernatants 

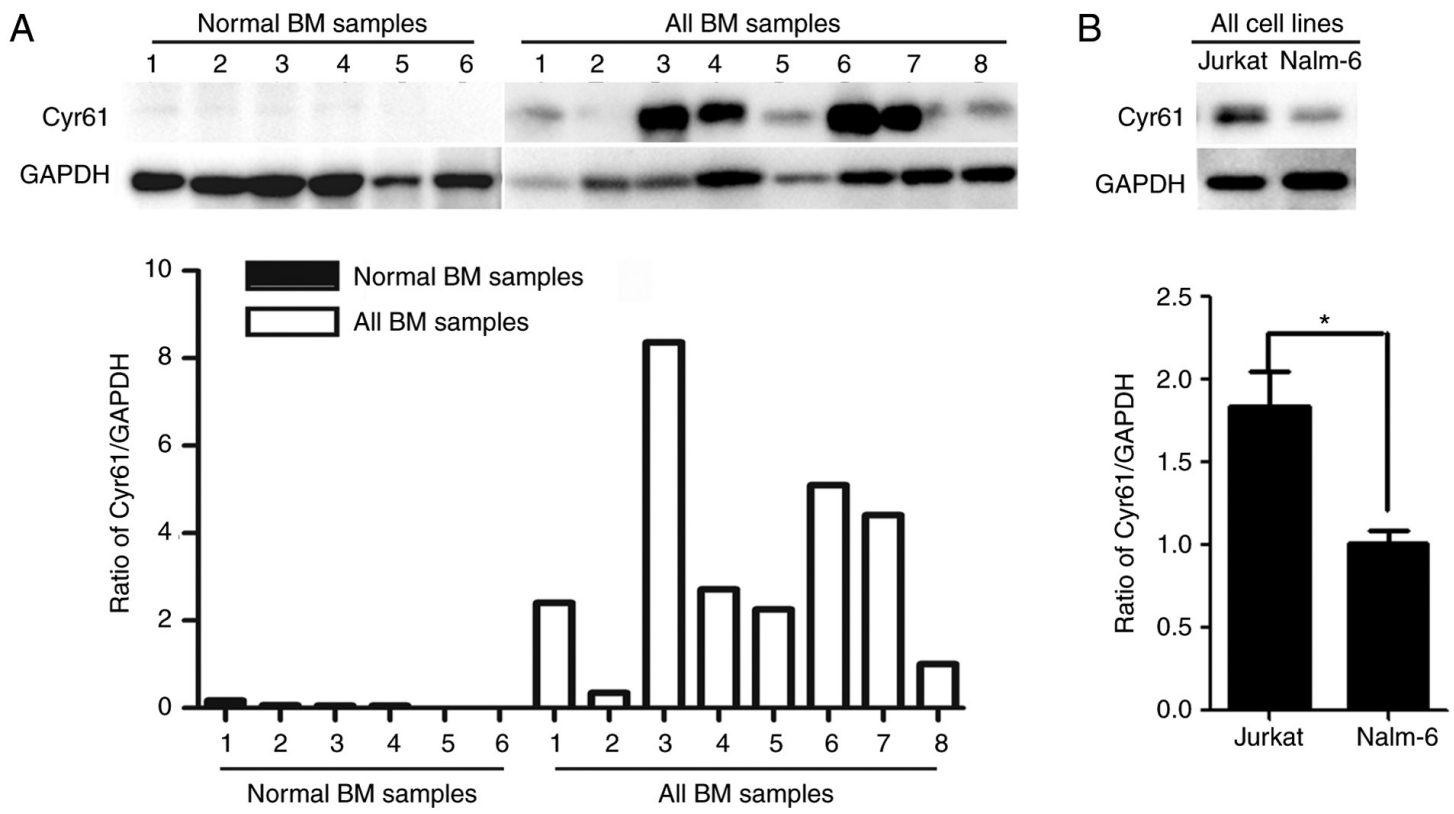

Figure 1. Level of Cyr61 expression in ALL cell lines and ALL bone marrow samples. (A) Cyr61 protein levels were determined in the BMMNCs derived from healthy donors and ALL samples by western blotting, and the ratio of Cyr61/GAPDH in the last ALL BM sample was taken as the control, and the ratio of Cyr61/GAPDH was set as 1, to calculate the relative expression of Cyr61 in other samples. (B) The level of Cyr61 protein was determined in two ALL cell lines (Jurkat and Nalm-6) by western blotting, and the ratio of Cyr61/GAPDH in Nalm-6 cells was taken as the control, in which the ratio of Cyr61/GAPDH was set as 1, to calculate the relative expression of Cyr61 in Jurkat cells. ${ }^{*} \mathrm{P}<0.05$. Cyr61, cysteine-rich 61; ALL, acute lymphoblastic leukemia; BMMNCs, bone marrow mononuclear cells; BM, bone marrow; GAPDH, glyceraldehyde-3-phosphate dehydrogenase.

from newly diagnosed patients with ALL with no treatment. Cell cultures were subjected to Ara-C treatment in the presence or absence of an anti-Cyr61 monoclonal antibody (mAb) 093G9. As shown in Fig. 2A, anti-Cyr61 monoclonal antibody (mAb) 093G9 could increase the Ara-C-induced Jurkat and Nalm-6 cell apoptosis, suggesting that the endogenous Cyr61 from ALL patient BM could decrease ALL cell apoptosis induced by Ara-C. Next, Jurkat and Nalm-6 cells were transiently transfected with a Cyr61-expressing plasmid (PEGFP-Cyr61) to overexpress Cyr61 in ALL cells. Transfection with PEGFP-Cyr61 significantly increased the level of Cyr61 protein in both Jurkat and Nalm-6 cells (data not shown). As expected, Ara-C-induced apoptosis was significantly decreased in Cyr61-overexpressing (PEGFP-Cyr61) ALL cells (Fig. 2B), suggesting that autocrine secretion of Cyr61 could confer resistance to Ara-C-induced apoptosis. To further address Cyr61's effect on the chemosensitivity of ALL cells, Jurkat and Nalm-6 cells were treated with exogenous Cyr61 (rhCyr61) at different concentrations, and cellular sensitivity to Ara-C-induced apoptosis was subsequently examined. As shown in Fig. 2C, exogenous Cyr61 also significantly downregulated the level of Ara-C-induced apoptosis in Jurkat and Nalm- 6 cells in a dose-dependent manner. Then, Jurkat and Nalm- 6 cells were treated with an anti-Cyr61 monoclonal antibody 093G9 to block rhCyr61 function, and the apoptosis induced by Ara-C were analyzed. The results showed that exposure to 093G9 significantly sensitized both Jurkat and Nalm- 6 cells to Ara-C treatment, resulting in increased apoptosis (Fig. 2D). Taken together, these results suggest that Cyr61 has an important role in the resistance of ALL cells to Ara-C.

Exogenous Cyr61 upregulates Bcl-2 in Ara-C-treated ALL cells. Since apoptosis is one of the key mechanisms of cytotoxicity mediated by chemotherapeutic drugs, the proteins of the Bcl-2 family have important roles in the regulation of apoptosis $(27,28)$. Thus, to investigate the underlying mechanism of Cyr61 induced drug resistance, real-time PCR and western blotting were used to analyze whether Cyr61 has an effect on Bcl-2 family molecules (Bcl-2, BCL-xL, Bax and XIAP) in Ara-C-treated ALL cells. The result showed that exogenous Cyr61 up-regulated the level of Bcl-2 mRNA in Ara-C-treated ALL cells (Fig. 3A, Jurkat cells, $\mathrm{P}<0.05$, Nalm-6 cells, $\mathrm{P}<0.05$, Student's t-test), but had little effect on BCL-xL, Bax, and XIAP mRNA expression. Additionally, it was found that exogenous Cyr61 could increase the level of Bcl-2 protein in Ara-C-treated ALL cells (Fig. 3B, Jurkat cells, $\mathrm{P}<0.05$, Nalm- 6 cells, $\mathrm{P}<0.05$, calculated by Student's t-test). Together, these results suggest that the existence of exogenous Cyr61 can desensitize Ara-C treatment of ALL cells, likely through upregulating Bcl-2 levels.

Exogenous Cyr61 decreases Ara-C-induced apoptosis through the $N F-\kappa B$ signaling pathway. Several reports have observed constitutive NF- $\kappa \mathrm{B}$ activation in ALL cells (29-31); this pathway could be activated by Cyr61, resulting in cellular proliferation and chemotherapy resistance in ovarian and breast cancer cells. To determine whether the NF- $\mathrm{B}$ pathway is involved in the anti-apoptotic function of Cyr61 in Ara-C-treated ALL cells, we evaluated the profile of the NF- $\mathrm{B}$ pathway using known inhibitors of this pathway, including PDTC (an inhibitor of $\mathrm{NF}-\kappa \mathrm{B}$ activation). The results showed that PDTC could significantly increase Ara-C-induced apoptosis in Cyr61-treated ALL cells and PDTC alone have no effect on the apoptosis of ALL cells (Fig. 4A). Moreover, Cyr61 treatment led to a dramatic increase in the phosphorylation of NF- $\kappa \mathrm{B}$ but not its overall expression in both Jurkat and Nalm-6 cells (Fig. 4B), indicating Cyr61 could activate NF- $\kappa$ B pathway in Jurkat and Nalm-6 
A Jurkat $B M+\lg G$
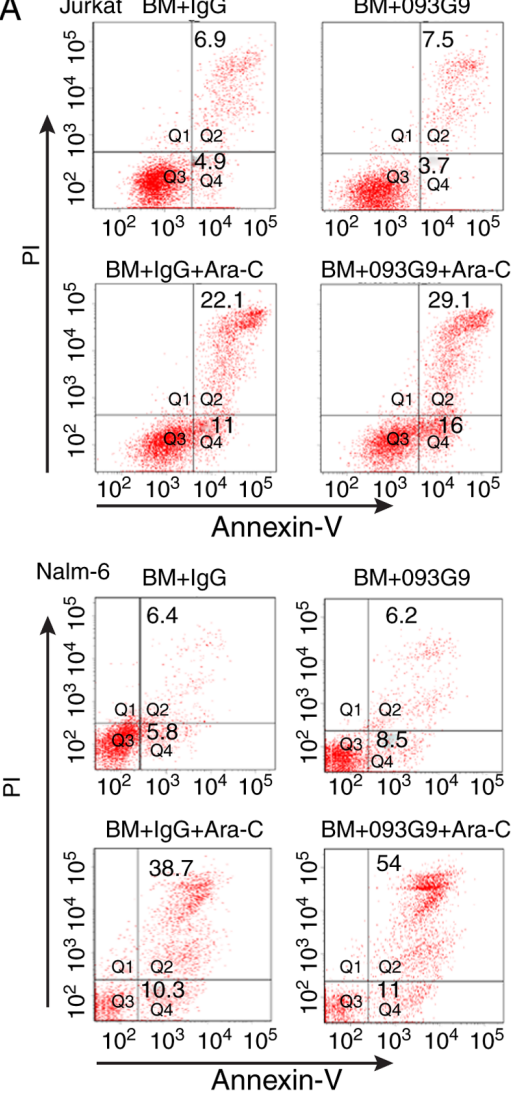
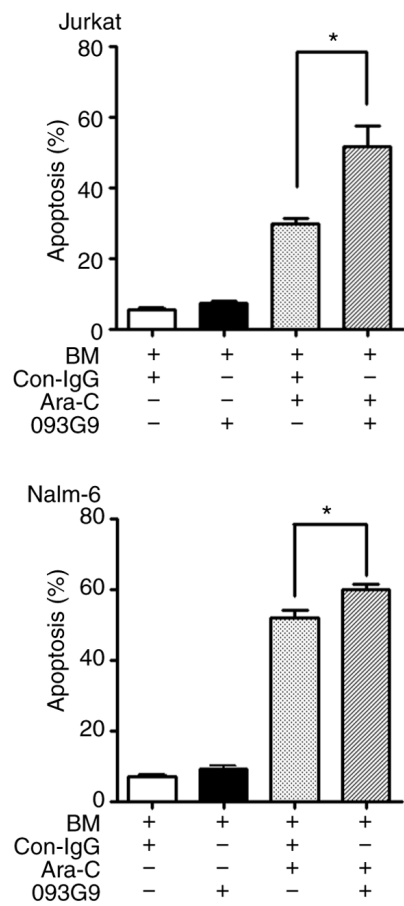
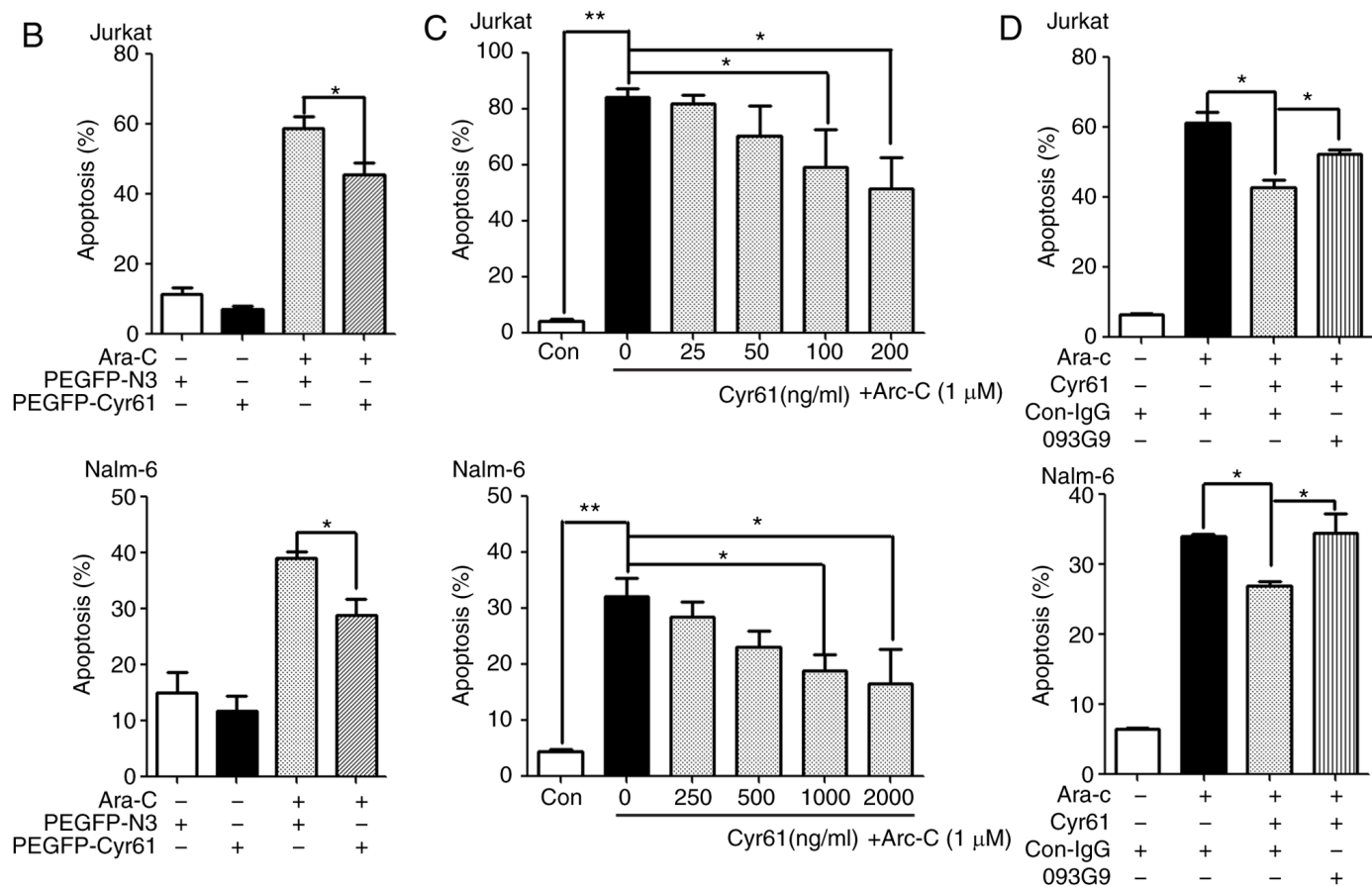

Figure 2. Cyr61 effectively decreases Ara-C-induced apoptosis in ALL cells. (A) Jurkat and Nalm-6 cells were incubated in BM supernatants (Cyr61 concentration is $185 \mathrm{pg} / \mathrm{ml}$ ) from patients with ALL with or without 1,000 pg/ml anti-human Cyr61 monoclonal antibody (093G9) for $24 \mathrm{~h}$, followed by exposure to $1 \mu \mathrm{M}$ Ara-C. After incubation for $24 \mathrm{~h}$, cells were collected and stained with Annexin V-FITC/PI and the percentages of apoptotic cells (FITC-positive) were measured using flow cytometric analysis. A murine isotype-matched antibody (con-IgG) served as a control. (B) Jurkat and Nalm-6 cells were transfected with a Cyr61 expression plasmid (PEGFP-Cyr61) and an empty plasmid (PEGFP-N3) for $48 \mathrm{~h}$ followed by exposure to $1 \mu \mathrm{M}$ Ara-C for $24 \mathrm{~h}$. Cells were then collected and stained with Annexin V-PE. The percentages of apoptotic cells (PE-positive) were measured with e flow cytometric analysis. (C) Jurkat and Nalm- 6 cells were pre-incubated with increasing concentrations of rhCyr61 for $24 \mathrm{~h}$, followed by exposure to $1 \mu \mathrm{M}$ Ara-C for $24 \mathrm{~h}$. Cell apoptosis was determined by flow cytometric analysis using Annexin V-FITC /PI double staining. (D) Cyr61-decreased apoptosis of Ara-C-induced ALL cells was restored by 093G9. rhCyr61 (100 ng/ml Cyr61 in Jurkat; $1,000 \mathrm{ng} / \mathrm{ml}$ Cyr61 in Nalm-6) were pre-incubated with a mouse anti-Cyr61 mAb (093G9) (500 ng/ml in Jurkat; $5,000 \mathrm{ng} / \mathrm{ml}$ in Nalm-6) for $2 \mathrm{~h}$ prior to adding to cell culture. A murine isotype-matched antibody (con-IgG) served as a control. After incubation for $24 \mathrm{~h}$, Jurkat and Nalm- 6 cells were treated with $1 \mu \mathrm{M}$ Ara-C for another $24 \mathrm{~h}$. Cell apoptosis was analyzed using an Annexin V-FITC/PI double-staining kit. Data are expressed as the mean percentage of apoptotic cells \pm SEM of at least three independent experiments in triplicate. ${ }^{*} \mathrm{P}<0.05,{ }^{* *} \mathrm{P}<0.01$. Cyr61, cysteine-rich 61 ; ALL, acute lymphoblastic leukemia; Ara-C, cytosine arabinoside; PI, propidium iodide; GAPDH, glyceraldehyde-3-phosphate dehydrogenase. 

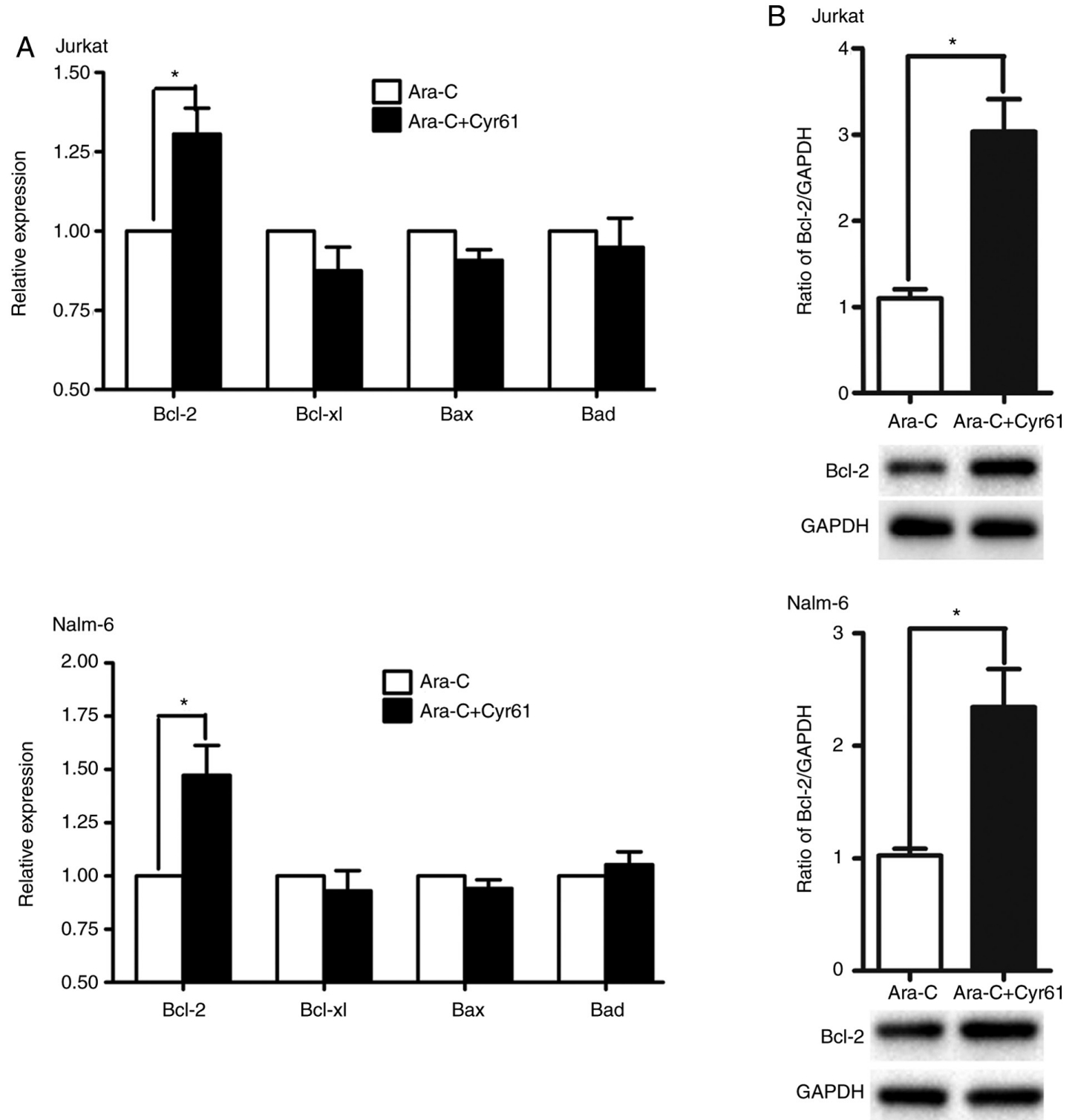

Figure 3. Exogenous Cyr61 upregulates Bcl-2 in Ara-C-treated ALL cells. (A) Jurkat and Nalm-6 cells were co-treat with Cyr61 (100 ng/ml for Jurkat cells; $1,000 \mathrm{ng} / \mathrm{ml}$ for Nalm- 6 cells) and $1 \mu \mathrm{M}$ Ara-C for $8 \mathrm{~h}$, and Bcl-2, BCL-xL, Bax, and XIAP mRNA expression were examined by real-time PCR. The expression levels of target genes were calculated with the $2^{-\Delta \Delta \mathrm{Ct}}$ method, employing GAPDH as an internal control. (B) Jurkat and Nalm- 6 cells were pre-incubated $(24 \mathrm{~h})$ with Cyr61 (100 ng/ml for Jurkat cells; $1,000 \mathrm{ng} / \mathrm{ml}$ for Nalm-6 cells) followed by addition with $1 \mu \mathrm{M}$ Ara-C for $24 \mathrm{~h}$. The relative level of Bcl-2 protein was examined by western blotting with the indicated antibodies, and the Ara-C-treated group was taken as the control sample, in which the ratio of $\mathrm{Bcl}-2 / \mathrm{GAPDH}$ was set as 1, to calculate the relative expression of Bcl-2. Data are expressed as the mean $\pm \mathrm{SEM}$ of at least three independent experiments in triplicate. ${ }^{*} \mathrm{P}<0.05$. Cyr61, cysteine-rich 61; ALL, acute lymphoblastic leukemia; Ara-C, cytosine arabinoside; Bcl-2, B-cell lymphoma-2; BCL-xL, B-cell lymphoma-extra large; Bax, Bcl-2-associated X protein; XIAP, X-linked inhibitor of apoptosis protein; GAPDH, glyceraldehyde-3-phosphate dehydrogenase.

cells. Taken together, these results suggest that Cyr61 decreases Ara-C-induced apoptosis via the $\mathrm{NF}-\kappa \mathrm{B}$ pathway.

Ara-C treatment increases the production of Cyr61 in ALL cells. To explore whether Ara-C can affect the production of Cyr61 in ALL cells, Jurkat and Nalm-6 cells were exposed to Ara-C for $24 \mathrm{~h}$. Western blotting was used to analyze the expression profiles of Cyr61 in ALL cells. As shown in Fig. 5, Ara-C could increase the production of Cyr61 in ALL cells.

\section{Discussion}

In this study, we found that Cyr61 was overexpressed in ALL BMMNCs. Furthermore, we observed that Cyr61 effectively decreased Ara-C-induced apoptosis in ALL cells, indicating chemoprotective effects of Cyr61 in ALL treatment.

Cyr61 is a secreted ECM protein, which is not only important for cell proliferation, survival, and migration, but also drug resistance in various tumors (12). It was recently reported that the level of Cyr61 is increased in BM supernatants from patients with ALL, and this change could promote ALL cell survival (22). Previous studies showed that BM stromal cells are the major source of Cyr61 $(21,32)$. Our current study showed that Cyr61 was overexpressed not only in the BMMNCs from patients with ALL, but also two ALL cell lines. It is speculated that, in addition to stromal cells, ALL cells could also be one of the sources generating Cyr61 in the bone marrow in an autocrine manner. 
A Jurkat
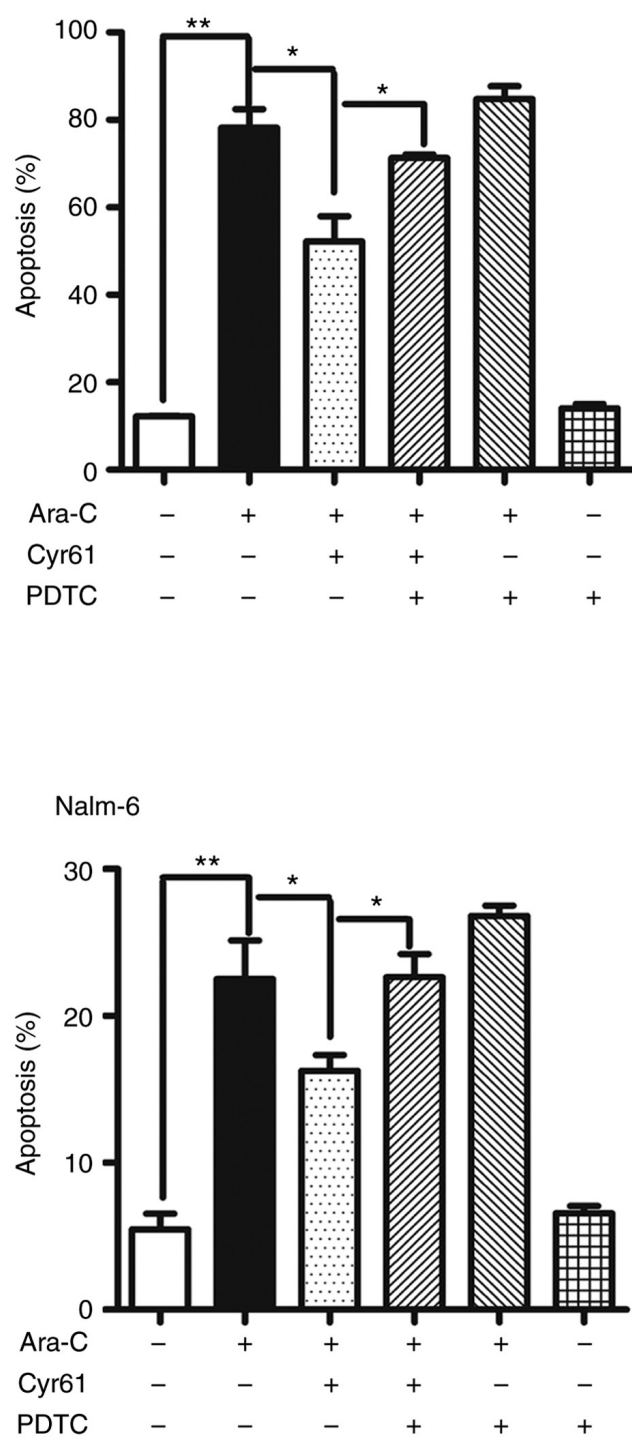

B
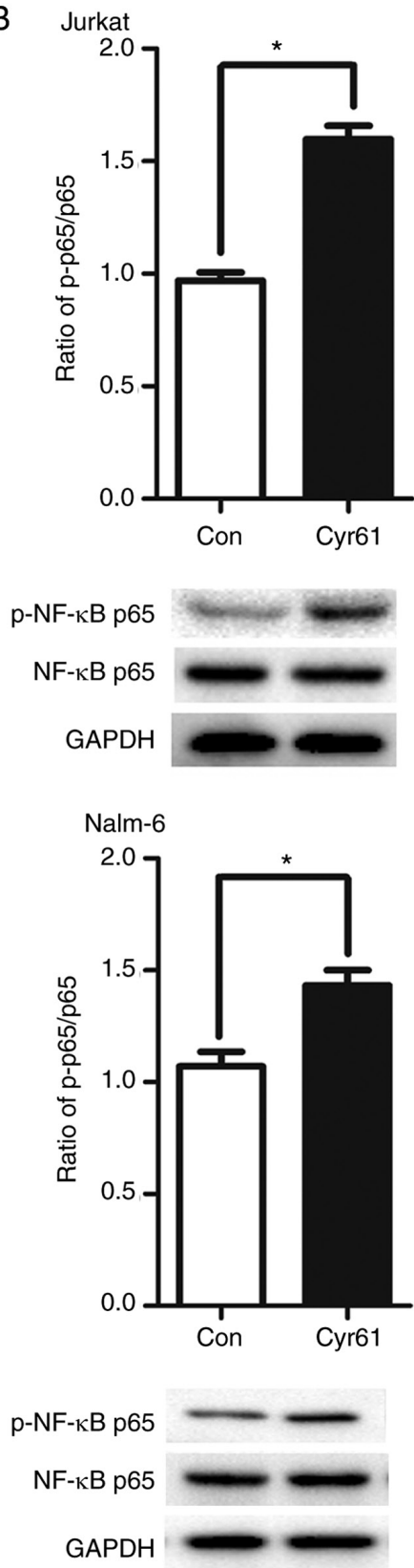

Figure 4. Exogenous Cyr61 decreases Ara-C-induced apoptosis through the NF- $\mathrm{B}$ signaling pathway. (A) Jurkat and Nalm-6 cells were pre-incubated with $4 \mu \mathrm{M}$ PDTC in association with Cyr61 (100 ng/ml for Jurkat cells; $1,000 \mathrm{ng} / \mathrm{ml}$ for Nalm-6 cells) for $24 \mathrm{~h}$ and then exposure to $1 \mu \mathrm{M}$ Ara-C for $24 \mathrm{~h}$. Cell apoptosis was analyzed using an Annexin V-FITC/PI double-staining kit. (B) Jurkat and Nalm-6 cells were incubated with Cyr61 (100 ng/ml for Jurkat cells; $1,000 \mathrm{ng} / \mathrm{ml}$ for Nalm- 6 cells) for $10 \mathrm{~min}$, and overall expression and phosphorylation of NF- $\mathrm{B}$ were examined by western blotting. The untreated group was taken as the control sample, in which the ratio of $\mathrm{Cyr} 61 / \mathrm{GAPDH}$ was set as 1 , to calculate the relative phosphorylation of $\mathrm{NF}-\kappa \mathrm{B}$. Data are expressed as the mean \pm SEM of at least three independent experiments in triplicate. ${ }^{*} \mathrm{P}<0.05,{ }^{* *} \mathrm{P}<0.01$. Cyr61, cysteine-rich 61 ; ALL, acute lymphoblastic leukemia; Ara-C, cytosine arabinoside; PDTC, ammonium pyrrolidinedithiocarbamate; GAPDH, glyceraldehyde-3-phosphate dehydrogenase.

Although multi-agent chemotherapy regimens are highly effective for patients with ALL, some responding patients eventually became refractory to initial therapy (3). Resistance to chemotherapeutic agents is a significant clinical problem for the successful treatment of leukemia. More studies have shown that the BM microenvironment contributes to leukemia cell resistance to chemotherapeutic agents. However, no study has yet explored the role of Cyr61 in ALL drug resistance, to the best of our knowledge.

Induction of apoptosis is a critical mechanism of cytotoxicity mediated by chemotherapeutic drugs, and resistance to apoptosis is a major obstacle in chemotherapy treatment $(33,34)$.
In the present study, it was found that BM-derived Cyr61 could decrease Ara-C-induced apoptosis in Jurkat and Nalm- 6 cells, and forced overexpression of Cyr61 in ALL cells enhanced their resistance to Ara-C-induced apoptosis, possibly through the autocrine secretion of Cyr61 into the microenvironment. In addition, it was observed that recombinant human Cyr61 increased the resistance of ALL cells to Ara-C, and that this effect was antagonized by the anti-Cyr61 antibody 093G9. Furthermore, it was observed that Jurkat cells (T-ALL cell lines) were more sensitive to the chemoprotective effects of Cyr61 than Nalm-6 cells (B-ALL cell lines), indicating that Cyr61 has differential chemoprotective effects on diverse cell types. These 
findings demonstrate, for the first time, that Cyr61 decreases the sensitivity of ALL cells to Ara-C, and that inhibition of the bioactivity of Cyr61 restores ALL cell response to Ara-C. The findings reported herein are consistent with previous results that Cyr61 decreases the apoptosis of tumor cells, leading to chemotherapy resistance in breast cancer, ovarian cancer and acute myeloid leukemia $(21,25,26)$. Therefore, Cyr61 may be one of the factors leading to drug resistance of ALL, and blocking pathways involved with Cyr61 function could be used for treating relapsed ALL. These results provide further evidence that BM microenvironment-derived soluble factors have important roles in the development and therapeutic response of leukemia cells.

It is well known that the Bcl-2 family of proteins are important apoptosis regulators that have essential roles in the apoptosis induced by chemotherapeutic drugs $(27,28)$. To investigate the mechanism underlying Cyr61-induced drug resistance, the influence of exogenous Cyr61 on the expression of Bcl-2, BCL-xL, $\mathrm{Bax}$, and XIAP was evaluated as a possible mechanism for Cyr61-induced Ara-C resistance. The results showed that Cyr61 could increase $\mathrm{Bcl}-2$ production without affecting the expression levels of BCL-xL, Bax, and XIAP in Ara-C-treated ALL cells. Considering that $\mathrm{Bcl}-2$ is an anti-apoptotic protein able to inhibit apoptosis, these findings suggest that the Bcl-2 pathway is involved in Cyr61-induced Ara-C resistance of ALL cells. Furthermore, it was found that Cyr61-induced $\mathrm{Bcl}-2$ production is higher in Jurkat cells than in Nalm-6 cells, which may be one of the reasons why Jurkat cells were more sensitive to Cyr61 chemoprotective effects than Nalm- 6 cells.

Previous studies have shown that NF- $\kappa \mathrm{B}$ is activated downstream of Cyr61, conferring malignant cell resistance to chemotherapy $(17,18)$. As expected, the NF- $\mathrm{KB}$ pathway also contributed to Cyr61-mediated ALL cell resistance to Ara-C. The findings reported herein are consistent with those of several previous reports in which Cyr61 activates the NF- $\mathrm{kB}$ signaling pathway, and subsequently confers resistance to certain chemotherapeutic drugs in breast cancer and ovarian cancer $(17,18)$. Numerous studies have demonstrated that the Bcl-2 family and NF- $\kappa \mathrm{B}$ proteins are closely associated with cell apoptosis (35-37). Notably, our previous studies found that the NF- $\kappa \mathrm{B}$ signaling pathway is involved in Cyr61-induced Bcl-2 production in ALL cells (22). On the basis of these results, NF- $\kappa \mathrm{B}$ proteins may be upstream controllers of Bcl-2 production in Cyr61-induced ALL cell resistance to Ara-C.

The ability of cells to counteract stressful conditions usually elicits the activation of pro-survival pathways and the production of molecules with antioxidant and anti-apoptotic activities to sustain cell survival. For example, Cyr61 is found to be markedly increased in prostate carcinoma PC-3 cells in response to $\mathrm{N}$-acetylcysteine induced cytotoxicity, and are beneficial for cell survival and anti-apoptosis under a cytotoxic microenvironment (19). In the present study, the results showed that Ara-C treatment markedly increased the levels of Cyr61 in Jurkat and Nalm-6 cells. Therefore, it is speculated that this is a part of the mechanism of ALL cell resistance to Ara-C. Thus, the significance and mechanism of Ara-C-induced Cyr61 expression need to be further studied.

There are several limitations to this study. First, the results rely solely on one chemotherapy drug (Ara-C). However, there are many alternative chemotherapeutic drugs commonly used for ALL treatment, such as vincristine, daunorubicin, and

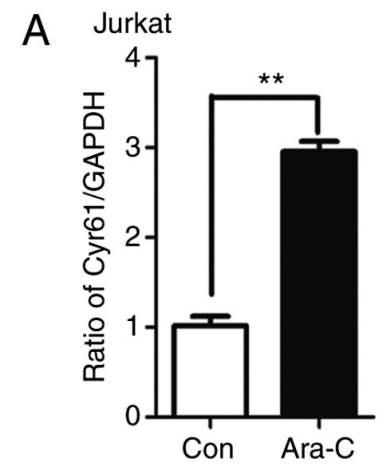

B Nalm-6
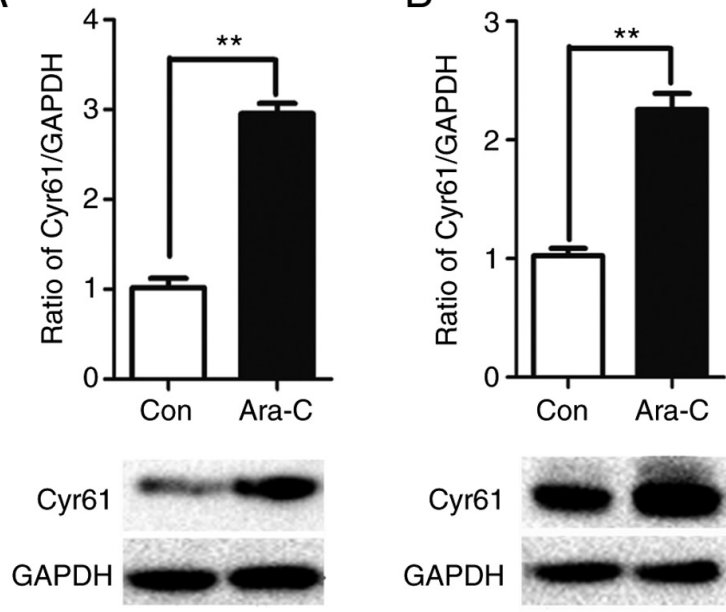

Figure 5. Ara-C treatment increases the production of Cyr61 in ALL cells. The protein levels (up) of Cyr61 in (A) Jurkat cells and (B) Nalm-6 cells treated with $1 \mu \mathrm{M}$ Ara-C for $24 \mathrm{~h}$ were detected using western blotting. The untreated group was used as the control sample, in which the ratio of Cyr61/GAPDH was set as 1 , to calculate the relative expression of Cyr61. Data are expressed as the mean \pm SEM of at least three independent experiments in triplicate. ${ }^{* *} \mathrm{P}<0.01$. Cyr61, cysteine-rich 61; ALL, acute lymphoblastic leukemia; Ara-C, cytosine arabinoside; GAPDH, glyceraldehyde-3-phosphate dehydrogenase.

dexamethasone. The role of Cyr61 in the chemosensitivity of ALL cells to other drugs remains unknown and needs to be elucidated. Second, the study on the Cyr61/NF- $\mathrm{\kappa B}$ signaling pathway was performed in vitro and thus lacks certain components of the BM microenvironment; further study should be conducted to elucidate the mechanism underlying Cyr61-mediated ALL cell resistance to Ara-C in vivo.

In the present study, the results showed that Cyr61 was highly expressed in BMMNCs from patients with ALL, and elevated Cyr61 levels conferred ALL cells with resistance to Ara-C-induced apoptosis, partially via the activation of the NF- $\kappa \mathrm{B}$ pathway. The present study indicates, for the first time, that Cyr61 may act as a chemoprotective factor for ALL cells, and that targeting Cyr61 directly or its relevant effector pathways might improve the clinical responses of patients undergoing treatment for ALL.

\section{Funding}

This study was supported by the Fujian Medical Innovation Project (grant no. 2017-CX-20), Fujian Province Joint Funds for the Innovation of Science and Technology (grant no. 2017Y9051), the Natural Science Foundation of Fujian Province (grant no. 2016J01569), the Training Project for Young and Middle-aged Core Talents in Health System of Fujian Province (grant nos. 2016-ZQN-31 and 2018-ZQN-69), the National Natural Science Foundation of China (grant no. 81700098), the Fujian Provincial Key Special Projects (grant nos. 2016Y9032 and 2016B041), the Construction Project of Fujian Medical Center of Hematology (grant no. Min201704), the National Key R\&D Program of China (grant no. 2016YFC0902800), the National Natural Science Foundation of China (grant no. 81470326), Fujian Provincial Public Health Project (grant no. WKJ2016-2-06), National and Fujian Provincial Key Clinical Specialty Discipline Construction Program, P. R.C 
(grant no. 2016-682) and the Fujian Provincial National Health and Family Planning Commission Project for Young (grant no. 2016-1-45).

\section{Availability of data and materials}

All data generated or analyzed during this study are included in this published article.

\section{Authors' contributions}

$\mathrm{XZ}$ and $\mathrm{JH}$ conceived the research and performed overall supervision in the study. YC, CW, XZ, YS, ZL, YK, PL, CZ, $\mathrm{QH}$ and TH performed the experiments. XZ, CW, YS, YC and $\mathrm{JH}$ performed data analysis. $\mathrm{XZ}, \mathrm{JH}, \mathrm{YC}$ and $\mathrm{CW}$ wrote the manuscript. $\mathrm{XZ}, \mathrm{JH}, \mathrm{YC}$ and $\mathrm{CW}$ contributed to the discussion of results and to the review of the manuscript. All authors read and approved the final manuscript.

\section{Ethics approval and consent to participate}

These studies were performed in accordance with the ethical guidelines under the protocols approved by the Institutional Medical Ethics Review Board of the Affiliated Union Hospital of Fujian Medical University, Fuzhou, China.

\section{Patient consent for publication}

Not applicable.

\section{Competing financial interests}

The authors declare no competing financial interests.

\section{References}

1. Pui CH: Childhood leukemias. N Engl J Med 332: 1618-1630, 1995.

2. Pui CH and Evans WE: Acute lymphoblastic leukemia. N Engl J Med 339: 605-615, 1998.

3. Pui CH and Jeha S: New therapeutic strategies for the treatment of acute lymphoblastic leukaemia. Nat Rev Drug Discov 6: 149-165, 2007.

4. Rowe JM: Prognostic factors in adult acute lymphoblastic leukaemia. Br J Haematol 150: 389-405, 2010.

5. Mudry RE, Fortney JE, York T, Hall BM and Gibson LF: Stromal cells regulate survival of B-lineage leukemic cells during chemotherapy. Blood 96: 1926-1932, 2000.

6. Gibson LF: Survival of B lineage leukemic cells: Signals from the bone marrow microenvironment. Leuk Lymphoma 43: 19-27, 2002.

7. Konopleva $\mathrm{M}$ and Andreeff M: Targeting the leukemia microenvironment. Curr Drug Targets 8: 685-701, 2007.

8. Boyerinas B, Zafrir M, Yesilkanal AE, Price TT, Hyjek EM and Sipkins DA: Adhesion to osteopontin in the bone marrow niche regulates lymphoblastic leukemia cell dormancy. Blood 121: 4821-4831, 2013.

9. Meads MB, Hazlehurst LA and Dalton WS: The bone marrow microenvironment as a tumor sanctuary and contributor to drug resistance. Clin Cancer Res 14: 2519-2526, 2008.

10. Liu J, Masurekar A, Johnson S, Chakraborty S, Griffiths J, Smith D, Alexander S, Dempsey C, Parker C, Harrison S, et al: Stromal cell-mediated mitochondrial redox adaptation regulates drug resistance in childhood acute lymphoblastic leukemia. Oncotarget 6: 43048-43064, 2015.

11. Dosen-Dahl G, Munthe E, Nygren MK, Stubberud H, Hystad ME and Rian E: Bone marrow stroma cells regulate TIEG1 expression in acute lymphoblastic leukemia cells: Role of TGFbeta/BMP-6 and TIEG1 in chemotherapy escape. Int J Cancer 123: 2759-2766, 2008 .
12. Lau LF: CCN1/CYR61: The very model of a modern matricellular protein. Cell Mol Life Sci 68: 3149-3163, 2011.

13. Chuang JY, Yu NY, Chiang IP, Lai CH, Lin CD and Tang CH: Cyr61 increases matrix metalloproteinase- 3 expression and cell motility in human oral squamous cell carcinoma cells. J Cell Biochem 113: 1977-1986, 2012.

14. Lin BR, Chang CC, Chen LR, Wu MH, Wang MY, Kuo IH, Chu CY, Chang KJ, Lee PH, Chen WJ, et al: Cysteine-rich 61 (CCN1) enhances chemotactic migration, transendothelial cell migration, and intravasation by concomitantly up-regulating chemokine receptor 1 and 2. Mol Cancer Res 5: 1111-1123, 2007.

15. Fromigue O, Hamidouche Z, Vaudin P, Lecanda F, Patino A, Barbry P, Mari B and Marie PJ: CYR61 downregulation reduces osteosarcoma cell invasion, migration, and metastasis. J Bone Miner Res 26: 1533-1542, 2011.

16. Leask A: A sticky situation: CCN1 promotes both proliferation and apoptosis of cancer cells. J Cell Commun Signal 4: 71-72, 2010.

17. Lee KB, Byun HJ, Park SH, Park CY, Lee SH and Rho SB: CYR61 controls p53 and NF- $\kappa$ B expression through PI3K/Akt/mTOR pathways in carboplatin-induced ovarian cancer cells. Cancer Lett 315: 86-95, 2012.

18. Lin MT, Chang CC, Chen ST, Chang HL, Su JL, Chau YP and Kuo ML: Cyr61 expression confers resistance to apoptosis in breast cancer MCF-7 cells by a mechanism of NF-kappaB-dependent XIAP up-regulation. J Biol Chem 279: 24015-24023, 2004.

19. Lee YJ, Lee DM and Lee SH: Production of Cyr61 protein is modulated by extracellular acidification and PI3K/Akt signaling in prostate carcinoma PC-3 cells. Food Chem Toxicol 58: 169-176, 2013.

20. Hesler RA, Huang JJ, Starr MD, Treboschi VM, Bernanke AG, Nixon AB, McCall SJ, White RR and Blobe GC: TGF- $\beta$-induced stromal CYR61 promotes resistance to gemcitabine in pancreatic ductal adenocarcinoma through downregulation of the nucleoside transporters hENT1 and hCNT3. Carcinogenesis 37: 1041-1051, 2016.

21. Long X, Yu Y, Perlaky L, Man TK and Redell MS: Stromal CYR61 confers resistance to mitoxantrone via spleen tyrosine kinase activation in human acute myeloid leukaemia. Br J Haematol 170: 704-718, 2015.

22. Zhu X, Song Y, Wu C, Pan C, Lu P, Wang M, Zheng P, Huo R, Zhang C, Li W, et al: Cyr61 participates in the pathogenesis of acute lymphoblastic leukemia by enhancing cellular survival via the AKT/NF-кB signaling pathway. Sci Rep 6: 34018, 2016.

23. Livak KJ and Schmittgen TD: Analysis of relative gene expression data using real-time quantitative PCR and the 2 (-Delta Delta C(T)) method. Methods 25: 402-408, 2001.

24. Zhang Q, Wu J, Cao Q, Xiao L, Wang L, He D, Ouyang G, Lin J, Shen B, Shi Y, et al: A critical role of Cyr61 in interleukin-17-dependent proliferation of fibroblast-like synoviocytes in rheumatoid arthritis. Arthritis Rheum 60: 3602-3612, 2009.

25. Rho SB, Woo JS, Chun T and Park SY: Cysteine-rich 61 (CYR61) inhibits cisplatin-induced apoptosis in ovarian carcinoma cells. Biotechnol Lett 31: 23-28, 2009.

26. Lai D, Ho KC, Hao Y and Yang X: Taxol resistance in breast cancer cells is mediated by the hippo pathway component TAZ and its downstream transcriptional targets Cyr61 and CTGF. Cancer Res 71: 2728-2738, 2011.

27. Youle RJ and Strasser A: The BCL-2 protein family: Opposing activities that mediate cell death. Nat Rev Mol Cell Biol 9: 47-59, 2008.

28. Mirjolet JF, Barberi-Heyob M, Didelot C, Peyrat JP, Abecassis J, Millon R and Merlin JL: Bcl-2/Bax protein ratio predicts 5-fluorouracil sensitivity independently of p53 status. Br J Cancer 83: 1380-1386, 2000.

29. Kordes U, Krappmann D, Heissmeyer V, Ludwig WD and Scheidereit C: Transcription factor NF-kappaB is constitutively activated in acute lymphoblastic leukemia cells. Leukemia 14: 399-402, 2000.

30. Xue TY, Xu W, An Q, Wu Y, Xu CP and Zhang XY: Expression of nuclear transcription factor kappaB in childhood acute lymphoblastic leukemia and its significance. Zhongguo Shi Yan Xue Ye Xue Za Zhi 15: 767-771, 2007 (In Chinese).

31. Dos Santos NR, Ghezzo MN, da Silva RC and Fernandes MT: $\mathrm{NF}-\kappa \mathrm{B}$ in T-cell acute lymphoblastic leukemia: Oncogenic functions in leukemic and in microenvironmental cells. Cancers (Basel) 2: 1838-1860, 2010. 
32. Niu CC, Zhao C, Yang Z, Zhang XL, Pan J, Zhao C and Si WK: Inhibiting CCN1 blocks AML cell growth by disrupting the MEK/ERK pathway. Cancer Cell Int 14: 74, 2014

33. Anand S, Penrhyn-Lowe S and Venkitaraman AR: AURORA-A amplification overrides the mitotic spindle assembly checkpoint, inducing resistance to Taxol. Cancer Cell 3: 51-62, 2003.

34. Lowe SW and Lin AW: Apoptosis in cancer. Carcinogenesis 21: 485-495, 2000.

35. Chen G, Wang Y, Li M, Xu T, Wang X, Hong B and Niu Y: Curcumol induces HSC-T6 cell death through suppression of Bcl-2: Involvement of PI3K and NF- $\kappa \mathrm{B}$ pathways. Eur J Pharm Sci 65: 21-28, 2014.
36. González-Ramos R, Defrère $\mathrm{S}$ and Devoto L: Nuclear factor-kappaB: A main regulator of inflammation and cell survival in endometriosis pathophysiology. Fertil Steril 98: 520-528, 2012

37. Cao JP, Niu HY, Wang HJ, Huang XG and Gao DS: NF- $\kappa B$ $\mathrm{p} 65 / \mathrm{p} 52$ plays a role in GDNF up-regulating $\mathrm{Bcl}-2$ and $\mathrm{Bcl}-\mathrm{w}$ expression in 6-OHDA-induced apoptosis of MN9D cell. Int J Neurosci 123: 705-710, 2013 